\title{
QR Code: Challenges and Potentials for Services in Pos-Covid Era
}

Roberson Bolzan ${ }^{1}$, Paula V. Martins ${ }^{2}$ and Sílvia Fernandes ${ }^{2^{*}}$

${ }^{1}$ Faculty of Economics (FE), University of Algarve. Campus de Gambelas 8005-139 Faro, Portugal

${ }^{2}$ FCT \& Cinturs - Research Centre for Tourism, Sustainability and Well-being, University of Algarve, Campus de Gambelas 8005-139 Faro, Portugal

\section{Abstract}

The choice of this work arises from the interest in launching innovative services to target people. We sought to integrate a technology that could bring more innovation to a product/ service, namely due to mobile trends. Among some options considered, we chose the QR code technology. With the help of a strategic plan, we evaluate whether there is a market and propensity for its widespread use in Portugal. Then, an interactive prototype of the supporting platform is presented for validation by potential users. Most participants consider the idea interesting and promising. They suggested add-ons such as: more video functions and a greater variety of events and resources to apply it. Given the challenges in society, we forecast its application to the quick request for specialized information and/or resources by critical activities such as in health, tourism, etc.

\section{Introduction}

This work aims to approach a business idea, underlying the future launch of a start-up company. This project relates to the innovative offer of a service, culminating in presenting the prototype of a platform for sending video messages through QR code. It is called "Send and Surprise".

We also analyze some topics that should guide prototyping according to the market. This will give us an understanding to know if there are conditions for its adoption and use. Then, it culminates in the validation of the proposed service through an interactive prototype.

\section{Exploring QR Code}

The quick response $(\mathrm{QR})$ code has revolutionized the code barrier. It is an interesting technological breakthrough, which has transformed the way consumers interact with sellers, service providers and other consumers. This is increasingly important, due to new challenges to face such as this pandemic that affected all sectors and required mobile or online work. The QR code activates tags and directs them to links that can open webpages, videos, text messages, among other resources.

This technology was invented by the Japanese company DensoWave, a Toyota subsidiary, in 1994. The main goal was to facilitate the process of cataloging automobile components. It consists of highdensity bidimensional graphical images, like barcodes composed of digital squares instead of bars. The compounds of those squares come together to create codes that, in turn, host the data that will be verified by mobile devices [1].

QR code reader applications (apps), downloadable on mobile communication devices (mobile phones, tablets, etc.) require the action of a built-in camera to decode the data. This technology allows integrate such apps with other systems or objects. QR codes are barcodes that connect us quickly from physical objects to the digital world [1]. The use of QR code is free of any license as it is an open source, defined and published as an ISO standard in 2010. Then, in 2011, the QR code became commercial for the first time in the telecommunications' sector. Today, it has gained great popularity due to mobile technology, a decisive support for its proliferation.
In the beginning, the main objective was a code to be quickly interpreted by reading equipment. But after some time, other companies began to explore different ways of using QRs so that their commercial use became widespread all over the world. These codes are changing the way we interact with people and products (magazines, books, ads, events..). They have also changed the way content is created and delivered [2].

\section{QR Code and Its Applications}

In 2019 about 2.7 billion people used smartphones and an estimated $90 \%$ of the population will access high-speed internet in 2020. Smartphone updates have also added QR code scanning capabilities to the camera app, so these codes become an integral part of daily life. Even the latest android smartphones have made QR code scanning a native feature. Inspired by China, South Korea has also witnessed an impressive growth in the number of QR scans. India and US markets have also adopted QR to make payments and turn shopping into a unique experience [3].

Digital watermarking, a method to protect information, can be realized on QR codes. In watermark embedding methods, the embedding strength is important. Algorithms are developed to adjust the embedding strength, and the QR code can still be decoded by a general decoder [4].

The possible uses of QRs are almost infinite, as this technology is present in our daily lives in the most diverse services/products [5]. Due to its versatility, it is used in several fields such as: online banking, customer service management, medical assistance, security applications and integrated in print ads, products, smooth surfaces, etc. Another form of QR widely used is in invoices and other documents for paying services.

*Corresponding Author: Dr. Sílvia Fernandes, FE \& Cinturs - Research Centre for Tourism, Sustainability and Well-being, University of Algarve, Campus de Gambelas 8005-139 Faro, Portugal; E-mail: sfernan@ualg.pt

Citation: Bolzan R, Martins PV, Fernandes S (2020) QR Code: Challenges and Potentials for Services in Pos-Covid Era. Int J Comput Softw Eng 5: 161. doi: http:// dx.doi.org/10.15344/2456-4451/2020/161

Copyright: (C) 2020 Bolzan et al. This is an open-access article distributed under the terms of the Creative Commons Attribution License, which permits unrestricted use, distribution, and reproduction in any medium, provided the original author and source are credited. 
Futuristic stores and supermarkets, that operate without staff and queues, also use QR codes. Before entering the store, the buyer needs to create an account and then download the app, creating a personal QR (like a digital signature). Then, when entering the store, an electronic check-in is performed that identifies the personal QR and automatically pays the products purchased.

The following sections focus on the description of the proposed service, the idea presentation survey, its prototype and a validation interview.

\section{Proposed Service}

This project, "Send and Surprise", intends to be a start-up in the area of new technologies. It has a first purpose of interacting with customers through video message sending, among other possibilities. It allows an interaction between the offline/traditional (invitations, cards, gifts, etc.) and the online/modern (videos, digital, QR code, smartphone) on an individual and personalized way.

Through the suggested platform, the message is delivered in the form of a video, using a QR-code tag embedded in a chosen card or gift. This has a printed $\mathrm{QR}$, which can be visualized anywhere and anytime, through a mobile device with web access. To do it, you must access the Send and Surprise website and view (or download) the prepared video, where a QR-code is generated which can be printed on an object (or other items) of choice, available on the same website. Figure 1 illustrates the steps and processes involved.

The goal of Send and Surprise is not sending ordinary messages/ videos, as most of those we often send and receive through YouTube. These are special messages regarding events, special occasions with a high degree of personalization and creativity.

This idea, based on perceived flow [6], has lessons for the field of online shopping, retailing, marketing promotion, and consumer behavior. Embedded QR codes are helpful to share and influence people perception and satisfaction. Sharing information via QR code will influence people to engage and share information with others online [7]. And this has impacts on their lives and purchases.

\section{Idea Prototyping}

In computing, a prototype is a software model or pattern. In this first prototype of the proposed idea, an architecture based on screens (or 'scenes') could be defined. For each of these scenes, specific routines are defined.

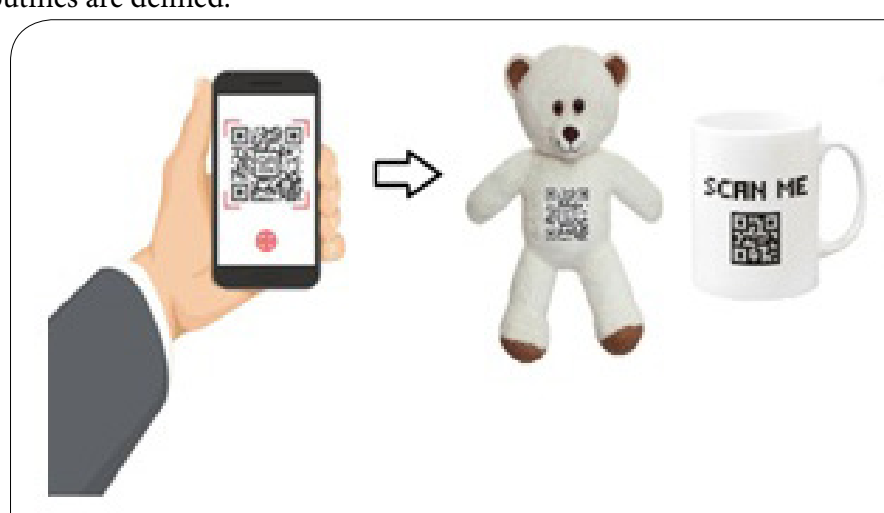

Figure 1: Sequence of the proposed app and service.
The validation interview has the main objective of knowing the opinion related with Send and Surprise application, especially to evaluate the prototype. It was an individualized interview, to users from the sample, in order to validate the functionality and clarity of the prototype.

The tests were carried out informally and in parallel with the development. Such tests focused basically on the interactivity of the application, verifying if the functionalities defined were executing as planned.

As this project links messages to QR codes which, subsequently direct people to a virtual store (in order to associate products to offer), it will be relevant to increase the storage capacity of a QR code. Cai et al. [8] propose an algorithm where modules for the padding codewords are modulated by the module-based binarized background image. Low-pass textured patterns are designed for both the black modules and the white modules. The module elimination parameter is optimized to maximize an objective function that accounts for higher visual quality and capacity. Besides these attributes, security is also crucial. That is why Cai et al. [9] developed an algorithm to decode a QR that allows, not only access to the relevant links embedded in it, but also restore the original image. For recovery of the QR code, it is bound with the background image. This increases its security level when users scan a QR code.

\section{Conclusion}

With new technologies, people have built a dependency of the mobile internet and its apps in daily life $[10,11]$. Therefore, the main objective of this work is to present an idea of a platform for sending video messages, through a $\mathrm{QR}$-code tag.

Data on the use of smartphones/e-commerce in Portugal justify market demand for this type of service proposed. To guide its implementation into the first prototype, some steps of the strategic plan were considered. Namely within the scope of QR-code technology, a survey was applied in order to capture the familiarity with it and the acceptance/adoption of the idea. Then, an interactive prototype was presented and an interview to some survey participants could give insight about it. The resulting answers help to outline further changes or innovations.

Regarding the survey, there is familiarity about QR codes having been used by most respondents. It is considered an easy-to-use technology by the majority of them. However, this interaction still occurs through conventional means such as product package,

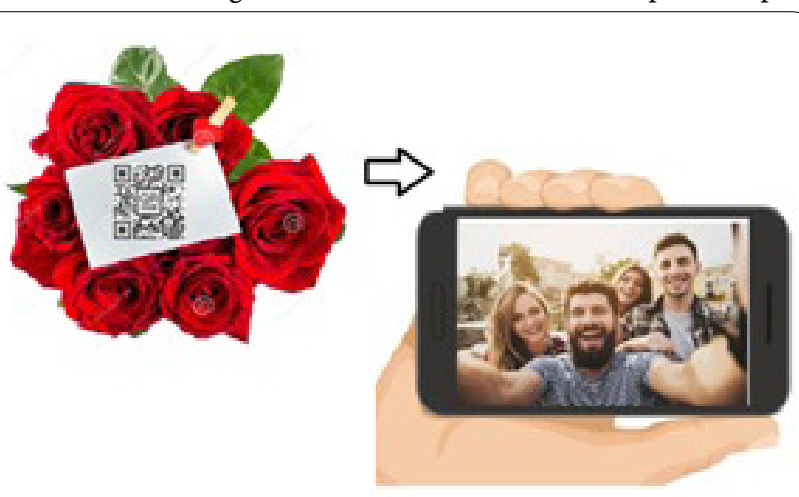


concert invitation and invoice payment. Regarding the interview for the prototype validation, participants find it easy to understand and interact. However, in its layout, they propose a change in color patterns and fonts for a more friendly navigation. They also suggested add-ons such as: more video functions and a greater variety of events and resources to apply it.

There is a promising scenario for the implementation of an e-service like Send and Surprise in the Portuguese market. Nevertheless, training aimed at promoting the effective use of QR-code technology must evolve.

Several goals underlying the development of Send and Surprise platform-prototype were achieved. However, there are still issues that can be developed to enrich it. In the future, we intend to extend it as a mobile app namely on Android, iOS and Windows Phone.

Given the present challenges, other possibilities to consider relate to its application to quick requests for information or resources by sectors such as health, security, engineering, etc.

\section{Competing Interests}

The authors declare that they have no competing interests.

\section{References}

1. Bashir I, Naik K, Madhavaiah C (2013) Potential business applications of Quick Response (QR) codes. Prajnan 41: 353-366.

2. Jharotia A (2018) QR codes: Innovative technology for libraries in electronic environment. In book: Essential Technologies for Libraries in Digital Era. Lambert Academic Publishing.

3. Beaconstac (2019) The Future of QR Codes: 2019 and Beyond.

4. Pan J, Sun X, Chu S, Abraham A, Yan B, et al. (2021) Digital watermarking with improved SMS applied for QR code. Engineering Applications of Artificial Intelligence.

5. Shin DH, Jung J, Chang BH (2012) The psychology behind QR codes: User experience perspective. Comput Hum Behav 28: 1417-1426.

6. Webster J, Trevino L, Ryan L (1993) The dimensionality and correlates of flow in human-computer interactions. Computers in Human Behavior 9: 411-426.

7. Hossain M, Zhou X, Rahman M (2018) Examining the impact of QR codes on purchase intention and customer satisfaction on the basis of perceived flow. International Journal of Engineering Business Management 10: 1-11.

8. Cai H, Yan B, Chen N, Yang HM (2019a) Beautified QR code with high storage capacity using sequential module modulation. Multimedia Tools and Applications 78: 22575-22599.

9. Cai H, Yan B, Pan J (2019b) Visual QR Code with beautication and selfrecovery based on data hiding. Genetic and Evolutionary Computing.

10. Dennison L, Morrison L, Conway G, Yardley L (2013) Opportunities and challenges for smartphone applications in supporting health behavior change: Qualitative study. J Med Internet Res 15: e86.

11. Hui S, Inman J, Huang Y, Suher J (2013) The effect of in-store travel distance on unplanned spending: Applications to mobile promotion strategies. Journal of Marketing 77: 1-16. 